\title{
BMJ Global Health BCG skin reactions by 2 months of age are associated with better survival in infancy: a prospective observational study from Guinea-Bissau
}

\author{
Frederik Schaltz-Buchholzer (D) ,1,2,3 Mike Berendsen (D) , 1,2,3,4 Adam Roth, 5,6 \\ Kristoffer Jarlov Jensen (D) , 1,7 Morten Bjerregaard-Andersen (D) ,,3 \\ Marcus Kjær Sørensen (D) ,2,3 Ivan Monteiro, ${ }^{2}$ Peter Aaby (D) ,2 \\ Christine Stabell Benn (1) 1,2,3,8
}

To cite: Schaltz-Buchholzer $\mathrm{F}$ Berendsen M, Roth A, et al. BCG skin reactions by 2 months of age are associated with better survival in infancy: a prospective observational study from Guinea-

Bissau. BMJ Global Health 2020;5:e002993. doi:10.1136/ bmjgh-2020-002993

Handling editor Alberto L Garcia-Basteiro

Received 24 May 2020 Revised 27 July 2020 Accepted 29 July 2020

\section{Check for updates}

(c) Author(s) (or their employer(s)) 2020. Re-use permitted under CC BY-NC. No commercial re-use. See rights and permissions. Published by BMJ.

For numbered affiliations see end of article.

Correspondence to MD, PhD Frederik SchaltzBuchholzer;

buchholzer@gmail.com

\section{ABSTRACT}

Introduction Receiving Bacille Calmette-Guérin (BCG)Denmark vaccine at birth has been associated with $40 \%$ reductions in all-cause neonatal mortality. We evaluated determinants of BCG skin reaction characteristics by age 2 months and tested the association with subsequent mortality. Methods Prospective observational study amalgamating five trials providing BCG-at-birth that were conducted between 2002 and 2018 in Guinea-Bissau. The reaction status and size were evaluated at home-visits by 2 months of age among 6012 neonates; mortality from 2 to 12 months was assessed at subsequent visits. Reaction determinants were evaluated by binomial regression providing risk ratios (RRs). In Cox-models providing adjusted mortality rate ratios (aMRRs), we assessed the association between (1) having a 2-month reaction (yes/no) and (2) reaction size tertiles and subsequent all-cause mortality risk. A subgroup had their BCG reaction evaluated and were bled at age 4 weeks; their samples underwent in vitro analysis for specific and non-specific cytokine responses.

Results The BCG strain was the main determinant for developing a 2-month reaction and the reaction size: the BCG-Russia/BCG-Denmark RR for large-reaction was 0.38 $(0.30-0.47)$ and the BCG-Russia/BCG-Japan RR was 0.61 (0.51-0.72). 5804 infants ( $96.5 \%$ ) were reactors by age 2 months; 208 (3.5\%) were non-reactors. The 2-12 months mortality risk was $4.8 \%(10 / 208)$ for non-reactors, $2.9 \%$ (64/2213) for small reactors, $1.8 \%$ (30/1710) for medium reactors and $0.8 \%$ (15/1881) for large reactors. The reactor/ non-reactor aMRR was $0.49(0.26-0.95)$ and there was a linear trend of decreasing mortality with increasing reaction size ( $\mathrm{p}$ for trend $<0.001$ ). BCG reactors had higher 4-week specific and non-specific cytokine responses, responses that were highest among those with large reactions.

Conclusion Among BCG-vaccinated infants, having a BCG skin reaction by age 2 months was associated with markedly better survival, as was the reaction size. Our findings thus support that BCG has substantial effects on all-cause mortality. Emphasising at-birth vaccination with immunogenic BCG strains and revaccinating non-reactors and small reactors could have major public health benefits.

Trial registration numbers NCT00146302, NCT00168610, NCT00625482, NCT01989026 and NCT02447536.

\section{Key questions}

What is already known?

- Vaccination with early-Bacille Calmette-Guérin (BCG)Denmark versus no-BCG has been associated with reduced neonatal mortality in randomised controlled trials.

- The BCG strain and the expertise of the vaccinator are the most important determinants for developing a BCG scar.

- Developing a BCG scar after BCG vaccination during infancy is associated with lower subsequent all-cause mortality.

What are the new findings?

- Among neonates that were BCG-vaccinated early (within 1 week after birth), developing a BCG reaction by 2 months of age was associated with substantially reduced mortality.

- The larger the size of the local BCG skin reaction by 2 months of age, the lower the subsequent mortality.

- Compared with BCG-Russia, the BCG-Denmark and BCG-Japan strains were associated with a higher incidence of BCG skin reactions and larger skin reactions by 2 months of age.

What do the new findings imply?

- BCG should be given at birth or as soon as possible after birth to ensure the development of early BCG reactions, and the vaccine should be administered by skilled vaccinators to ensure that large reactions develop.

- Certain immunogenic BCG strains that contain more viable mycobacteria are likely to induce more beneficial non-specific effects.

- Vaccination programmes should actively monitor BCG skin reaction kinetics among BCG-vaccinated infants, since many lives could be saved by increasing the focus on timely BCG vaccination, immunogenic BCG strains and vaccination quality.

\section{INTRODUCTION}

More than 120 million vaccine doses of Bacille Calmette-Guérin (BCG) against tuberculosis (TB) are administered every year, making 
BCG one of the world's most widely used vaccines. ${ }^{1}$ BCG vaccination has been associated with beneficial effects on all-cause mortality which are not explained by protection against $\mathrm{TB} .^{2-5}$ The recommended policy is vaccination at birth, but in practice vaccination is often delayed. Furthermore, a range of genetically divergent BCG strains are used interchangeably. In randomised controlled trials (RCTs) conducted in Guinea-Bissau among low-weight neonates, receiving BCG-Denmark at birth rather than the usual practice of delayed BCG was associated with a $38 \%$ (95\% Confidence Interval (CI): $17 \%$ to $54 \%$ ) reduction in neonatal mortality and BCGDenmark reduced the risk of fatal sepsis among hospitalised neonates by $54 \%$ (2\% to $78 \%))^{34}$ These marked beneficial effects could not be ascribed to prevention of TB. Hence, as other live vaccines, BCG appears to have beneficial non-specific effects (NSEs) inducing resistance towards unrelated infections. ${ }^{5}$ Epigenetic modifications of innate immune cells, known as trained innate immunity, has been suggested as an immunological pathway. ${ }^{6}$

Interestingly, observational studies indicate that among BCG-vaccinated infants, developing a BCG scar at the vaccine injection site is associated with a marked reduction in all-cause infant mortality. ${ }^{7-13}$ The main determinants for development of a BCG scar are vaccination technique and the BCG strain used. ${ }^{14-16}$ A small RCT from Australia has reported that the BCG-Denmark and BCG-Japan strains are associated with higher frequencies of mycobacteria-specific $\mathrm{T}$ cells, elevated Th1 cytokine secretion and larger skin reactions, when compared with BCG-Russia. ${ }^{17}$ Similarly, a recent large-scale RCT from Guinea-Bissau reported that BCG-Denmark and BCGJapan are associated with a higher BCG scar prevalence and larger scars when compared with BCG-Russia. ${ }^{15}$

It is unknown whether early BCG skin reactions and the size of these reactions affects mortality risk. We therefore extracted data from five RCTs conducted in GuineaBissau where BCG was provided shortly after birth. Among infants that had received BCG during the first week of life, we reviewed determinants for developing an early BCG reaction by 2 months of age and the reaction size and evaluated the association with the subsequent 2-12 month all-cause mortality risk.

\section{METHODS \\ Setting}

The Bandim Health Project (BHP, www.bandim.org) maintains a Health and Demographic Surveillance System (HDSS) covering approximately 100000 inhabitants in Bissau, the capital of Guinea-Bissau, a developing country with high infant mortality. The Hospital Nacional Simão Mendes (HNSM) is the country's main hospital facility and principal birthplace with 6000-7000 deliveries/year; it is located within a few kilometres from the Bandim HDSS. Since 2002, BHP has conducted a series of trials at HNSM evaluating the overall health effects of neonatal BCG vaccination. ${ }^{3}{ }^{15}{ }^{18-20}$ At HNSM,
BHP staff document all births and vaccinations with BCG and oral polio vaccine (OPV), which are exclusively provided by two experienced BHP vaccinators. In RCT $\mathrm{V}$, the post-vaccination weal size was measured to document the quality of the vaccination provided. Subsequent infant vaccines are provided at smaller health centres in accordance with the WHO-recommended immunisation schedule.

\section{Patient involvement}

This research was done without patient involvement. Patients were not invited to comment on the study design and were not consulted to develop patient relevant outcomes or interpret the results. Patients were not invited to contribute to the writing or editing of this document for readability or accuracy.

\section{Participants}

The eligibility criteria for infants to be included in the present observational study were: first, to have been enrolled in one of five BCG RCTs and have been BCGvaccinated by our team at either HNSM or a HDSS health centre during the first week of life (to allow time for a skin reaction to have developed at the BCG injection site by the visit at 2 months of age) and, second, to have received standardised home-visits as part of routine RCT procedures providing 2-month BCG skin reaction data. Mortality data up to 12 months of age was obtained through subsequent home-visits (table 1).

\section{Study design: the five BCG RCTs}

The five trials had the following study designs: RCT I, ${ }^{18}$ RCT $\mathrm{II}^{19}$ and RCT $\mathrm{III}^{3}$ shared the same study design, randomising healthy low-weight neonates to early BCG-Denmark versus delayed BCG. Only neonates randomised to early BCG and thus vaccinated by our team were included in the present study. RCT IV ${ }^{20}$ evaluated the effects of BCG on in-hospital nursery mortality, randomising neonates to receive immediate BCG upon nursery admission versus BCG at discharge. RCT $\mathrm{V}^{15}$ randomised neonates to different BCG vaccine strains; only the subgroup of infants that resided within the HDSS received home follow-up visits and were eligible for the present study. Both RCT IV and V were affected by a production halt at the BCG-Denmark manufacturer occurring in 2015, which lead to the substitution of BCGDenmark with BCG-Japan from July 2016 (table 1).

In all five RCTs, BHP staff vaccinated neonates by intradermal injection of $0.05 \mathrm{~mL}$ BCG in the left deltoid region, followed by oral administration of OPV. Following intradermal injection of BCG, a superficial post-vaccination weal remains visible on the skin for up to $30 \mathrm{~min} .{ }^{21}$ In RCT V, our two vaccinators assessed the size of the postvaccination weal immediately after vaccination. Between 2 and 4 weeks after vaccination, most infants develop a papule at the skin site where BCG was injected, and the usual trajectory is for this papule to transform to a pustule 


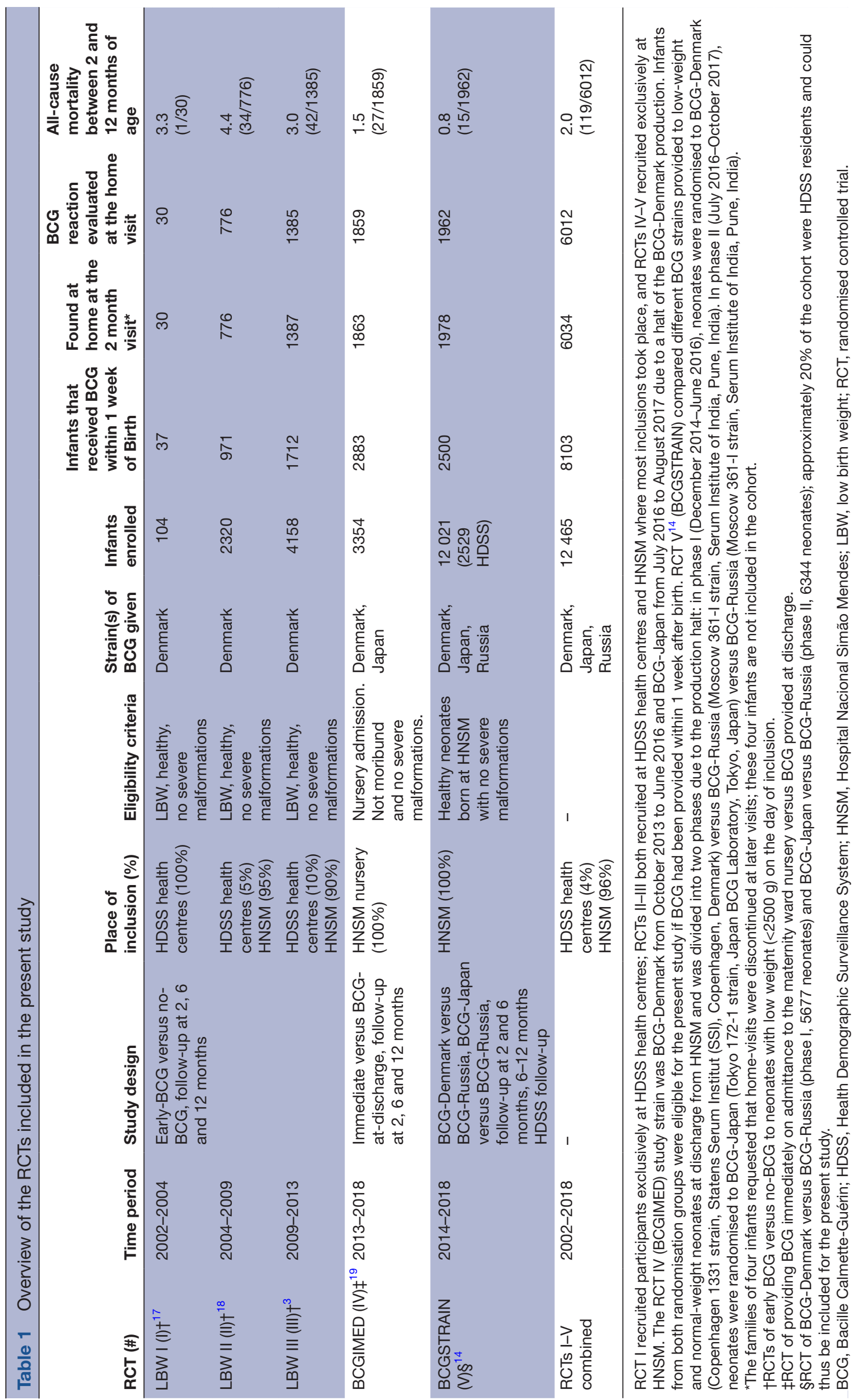




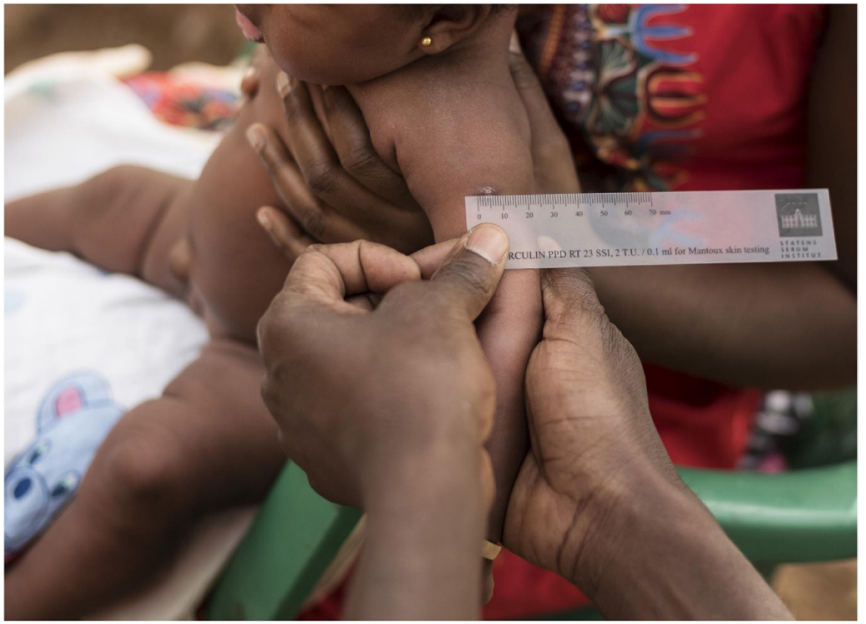

Figure 1 Measurement of an infant's Bacille CalmetteGuérin skin reaction at the vaccine injection site in the left deltoid region during a home-visit within the BCGSTRAIN randomised controlled trial (RCT V).

before healing within 2-5 months leaving a permanent superficial scar. $^{22}$

All RCTs had as exclusion criteria severe malformation and being moribund. Infants from families that refused our follow-up visits were excluded from analysis. The trials were conducted consecutively spanning the years 2002-2018, during which there was a country-wide trend of declining infant mortality. ${ }^{23}$

\section{Follow-up procedures}

BHP field assistants conducted standardised home-visits at 2, 6 and 12 months of age (RCTs I-IV) or at 2 and 6 months of age (RCT V). For RCT V, the 6-12 months mortality risk was obtained from the routine HDSS data collection. The HDSS provided address information used to locate HDSS infants, while BHP staff transported non-HDSS infants to their home after enrolment (RCTs I-IV); a map was drawn to ensure that the house could be located again. At the home-visits, the team collected anthropometric data including infant mid upper-arm circumference (MUAC) and weight, and assessed mortality, morbidity, and adverse events.

The assistant reviewed the infant's BCG skin reaction status by noting the presence or absence of a reaction (defined as a BCG papule, pustule or scar) at the vaccine injection site on the left upper deltoid and measured the horizontal and vertical reaction diameter using a transparent ruler (figure 1). Families are often absent or travelling; we therefore returned three times to each house and, if all visits were unsuccessful, information on survival was collected from those present. Infants whose family had moved were censored in the survival analysis from the date the family had moved.

\section{Immunological subgroup study}

As part of the RCT III trial procedures, a subgroup of infants was visited at home at 4 weeks of age and bled in order to compare BCG group versus control group immune responses, when few control group neonates had received BCG. Provided that informed consent was obtained, their BCG reaction was assessed and a capillary blood sample was collected for immunological assessment. ${ }^{24}$ A whole-blood differential count was performed on the EDTA-treated blood on an ABX Pentra60 (Horiba, France). In vitro stimulation with PMA and ionomycin, purified protein derivative (PPD) from Mycobacterium tuberculosis and different Toll-like receptor (TLR) agonists was performed on fresh heparinised blood, diluted 1:9 with RPMI-1640 and stimulated for 24 hours after which supernatants were harvested. Samples were stored and transported at $-70^{\circ} \mathrm{C}$ until analysis at Statens Serum Institut in Denmark, previously described in detail. $^{24}$ Cytokine concentrations of interleukin (IL)-1 $\beta$, IL-5, IL-6, IL-10, IL-17, interferon (IFN)- $\gamma$ and tumour necrosis factor (TNF)- $\alpha$ in the resulting supernatants were analysed by an immunobead-based multiplexed assay based on flowmetric Luminex xMAP technology.

\section{Statistical methods}

We tested determinants of 2-month BCG skin reactions and reaction sizes to calculate risk ratios (RRs) using binomial regression. The BCG reaction size was calculated as the measured height plus width divided by two. Tertiles of reaction sizes were computed using the Stata xtile command. Due to aggregation of reaction sizes, the approximated tertiles do not represent exactly $33 \%$ of the total.

The 2-12 months mortality risk by BCG reaction status at 2 months of age was assessed in crude and adjusted Cox proportional hazards models providing mortality rate ratios (MRRs) with age as the underlying time variable; age was thus inherently controlled for in all analyses. We computed cumulative mortality curves using the Kaplan-Meier estimate based on the date of the BCG reaction assessment and the date of death or censoring. We present crude MRRs stratified by RCT and adjusted MRRs (aMRRs) stratified by RCT and adjusted for maternal MUAC, year and the assistant that assessed the reaction at the 2-month home-visit, analysed by reaction status (yes/no) at 2 months of age and reaction size tertile (small, medium or large). Personyears-at-risk (Pyrs) were calculated from the date of the 2-month reaction assessment and infants contributed risk-time until they died, migrated or reached 12 months of age, whichever came first. Tests of proportionality of hazards were computed using Schoenfeld's residuals. We conducted a supplementary analysis evaluating cause of death data collected at the HNSM paediatric ward, at home-visits and from standardised verbal autopsies using the WHO/INDEPTH questionnaire. ${ }^{25}$ The verbal autopsies were reviewed by local physicians who assigned the cause of death and were blinded to the infant's BCG reaction status. The cause of death data was divided in infectious and non-infectious causes and analysed in Cox models providing aMRRs by 2-month reaction status and reaction size. We conducted a sensitivity analysis to test 


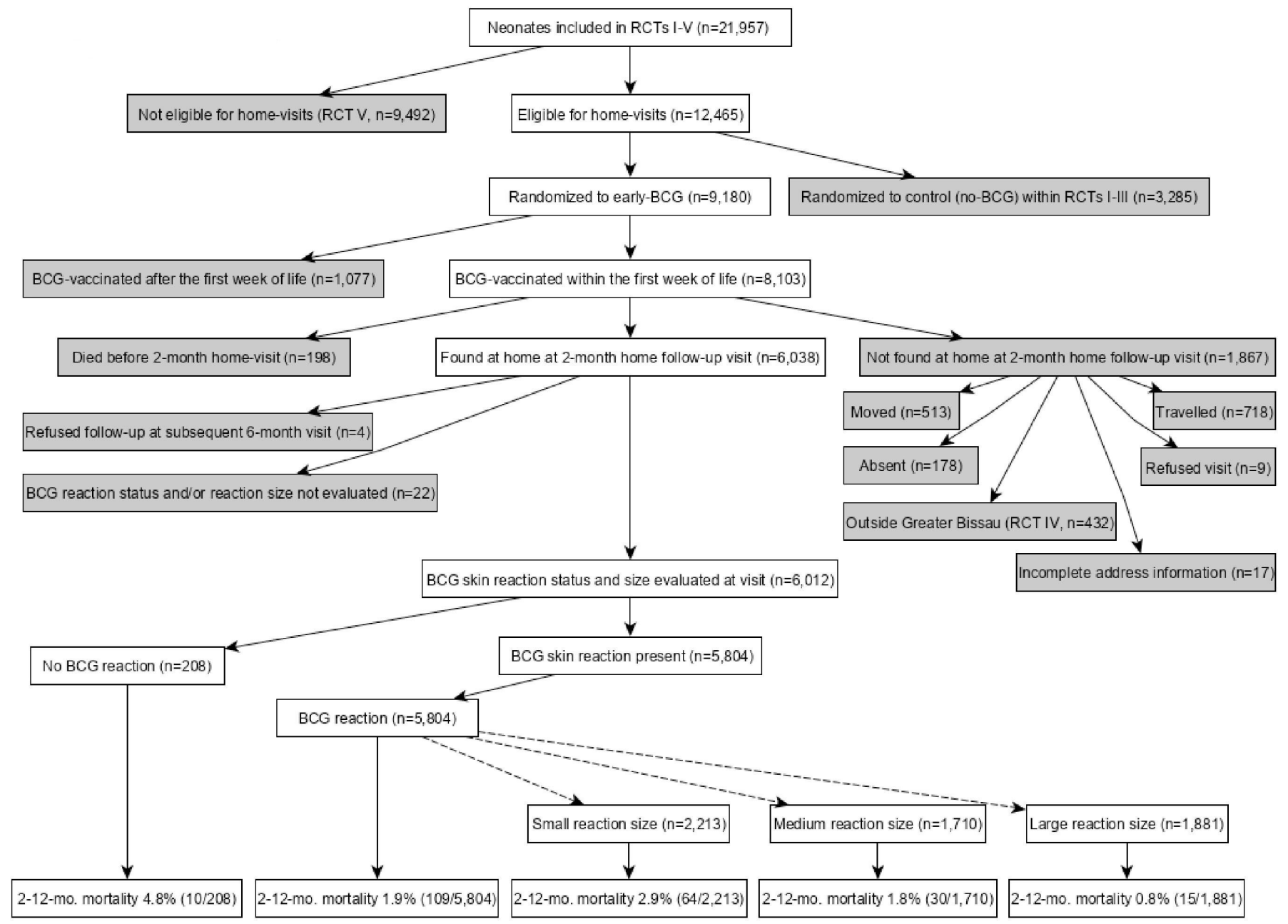

Figure 2 Study flow chart. BCG, Bacille Calmette-Guérin; RCT, randomised controlled trial.

whether assessor differences in 2-month BCG reaction assessments affected outcomes (online supplemental material). To analyse cytokine responses at 4 weeks of age among infants that were bled in RCT III, we compared log-transformed stimulated cytokine responses by 4-week reaction status (yes/no) and 4-week reaction size. Cytokine values that were either over or under the detection limit of the assay were imputed using multiple imputation $(\mathrm{n}=20)$ for censored interval variables when less than $50 \%$ of values were censored, as described by Uh et $a l .{ }^{26}$ When more than $50 \%$ of cytokine values were over the detection limit, values were dichotomised as detected or overdetection-limit. We assessed the data by linear regression or Poisson regression with robust variances adjusted for the non-stimulated response providing geometric mean ratios (GMRs) and prevalence ratios (PRs), respectively. All analyses were conducted overall and by sex using StataIC V.16 (Stata Corp) and all estimates are reported with $95 \%$ CIs.

\section{RESULTS}

The five RCTs included 21957 infants, 12465 of whom (RCT I: 104; II: 2320; III: 4158; IV: 3354; V 2529) were eligible for home-visits (figure 2). Of these, 9180 were randomised to receive early-BCG and 8103 of those were BCG-vaccinated during the first week of life. By 2 months of age, 198 infants had died and 1867 were not found at home (figure 2). Four families refused our subsequent 6-month visit and were excluded from the analysis. In summary, a total of 6034 infants who had received BCG within 1 week of birth were found at home at the 2-month visit and 99.6\% (6012/6034) of these had their BCG skin reaction evaluated. The median age at BCG vaccination was 1 day (online supplementary table 1); $76 \%(4551 / 6012)$ were vaccinated within the first 2 days of life. The 2-month BCG reaction prevalence was $97 \%(5804 / 6012) ; 37 \%(2213 / 6012)$ of the infants had a small reaction (median size $3.5 \mathrm{~mm}$, IQR 3.0-4.0), $28 \%(1710 / 6012)$ had a medium reaction $(5.0 \mathrm{~mm}$, IQR $4.5-5.0)$ and $31 \%(1881 / 6012)$ had a large reaction $(6.0$ mm, IQR 5.5-7.0) (table 2).

Pustules (open lesions) represented 48\% (2897/6012) of reactions with a median reaction size of $5.0 \mathrm{~mm}(4.0$ $6.0), 7.5 \%(451 / 6012)$ were papules with a median size of $4.5 \mathrm{~mm}(3.5-5.5)$ and $41 \%(2456 / 6012)$ were scars with a median size $4.0 \mathrm{~mm}(3.5-5.0)$. The median 2-month MUAC was $122 \mathrm{~mm}$ among non-reactors and $124 \mathrm{~mm}$ for reactors $(p<0.001)$. Among reactors, the median 
Table 2 Distribution of BCG skin reactions at the 2-month follow-up visit, median reaction size and all-cause mortality between 2 and 12 months, by RCT

\begin{tabular}{llllllll}
\hline RCT & $\begin{array}{l}\text { Proportion with } \\
\text { no skin reaction } \\
\%(\mathbf{n} / \mathbf{N})\end{array}$ & $\begin{array}{l}\text { Proportion with } \\
\text { a skin reaction } \\
\% \mathbf{n} / \mathbf{N}\end{array}$ & $\begin{array}{l}\text { Small reaction } \\
\%(\mathbf{n} / \mathbf{N})\end{array}$ & $\begin{array}{l}\text { Medium } \\
\text { reaction } \\
\%(\mathbf{n} / \mathbf{N})\end{array}$ & $\begin{array}{l}\text { Large } \\
\text { reaction } \\
\%(\mathbf{n} / \mathbf{N})\end{array}$ & $\begin{array}{l}\text { Median size* } \\
\text { of BCG skin } \\
\text { reaction }\end{array}$ & $\begin{array}{l}\text { Mortality between } \\
\mathbf{2} \text { and 12 months } \\
\%(\mathbf{n} / \mathbf{N})\end{array}$ \\
\hline I-III & $5(112 / 2191)$ & $95(2079 / 2191)$ & $45(978 / 2191)$ & $25(552 / 2191)$ & $25(549 / 2191)$ & $4.5(3.5-5.5)$ & $3.5(77 / 2191)$ \\
IV & $2(41 / 1859)$ & $98(1818 / 1859)$ & $32(602 / 1859)$ & $30(565 / 1859)$ & $35(651 / 1859)$ & $5.0(4.0-5.5)$ & $1.5(27 / 1859)$ \\
V & $3(55 / 1962)$ & $97(1907 / 1962)$ & $32(633 / 1962)$ & $30(593 / 1962)$ & $35(681 / 1962)$ & $5.0(4.0-5.5)$ & $0.8(15 / 1962)$ \\
RCTs I-V & $3(208 / 6012)$ & $97(5804 / 6012)$ & $37(2213 / 6012)$ & $28(1710 / 6012)$ & $31(1881 / 6012)$ & $4.5(4.0-5.5)$ & $2.0(119 / 6012)$ \\
combined & & & & & & & \\
\hline
\end{tabular}

*Height plus width divided by two among infants with a reaction, in millimetres. The median size of small reactions was $3.5 \mathrm{~mm}$ (IQR $3.0 \mathrm{~mm}-4.0$ $\mathrm{mm})$, for medium reactions it was $5.0 \mathrm{~mm}(4.5 \mathrm{~mm}-5.0 \mathrm{~mm})$ and for large reactions it was $6.0 \mathrm{~mm}(5.5 \mathrm{~mm}-7.0 \mathrm{~mm})$.

BCG, Bacille Calmette-Guérin; RCT, randomised controlled trial.

2-month MUAC was $122 \mathrm{~mm}$ for small-reactors, $124 \mathrm{~mm}$ for medium-reactors $(\mathrm{p}<0.001)$ and $128 \mathrm{~mm}$ for largereactors $(\mathrm{p}<0.001)$.

\section{Mortality risk}

We registered 119 deaths between the 2-month visit and 12 months of age; mortality declined from 3.5\% (77/2291) during the low-weight RCTs I-III to $0.8 \%$ $(15 / 1962)$ in RCT V, which included both low-weight and normal-weight children (table 2). The all-cause 2-12 month mortality across the trials was $4.8 \%$ (10/208) for non-reactors and $1.9 \%(109 / 5804)$ for reactors (table 3$)$, the corresponding crude reactor/non-reactor MRR stratified by RCT being $0.48(0.25-0.93)$ (table 4$)$ and the aMRR 0.49 (0.26-0.95) (figure 3).

By sex, the reactor/non-reactor aMRR was $0.42(0.15-$ 1.18 ) for male infants and $0.54(0.23-1.26)$ for female infants ( $p$ for same effect=0.70) (online supplemental table 2).

By reaction size, the 2-12 months mortality risk was $2.9 \%(64 / 2213)$ for infants with small reactions, $1.8 \%$ $(30 / 1710)$ for medium reactions and $0.8 \%(15 / 1881)$ for large reactions (table 3$)$. The large/small reaction aMRR was $0.35(0.20-0.63)$ (figure 4$)$. The association between reaction size and mortality was consistent

Table 3 Mortality risk between 2 and 12 months by 2-month BCG reaction status, reaction size and strain of BCG $B C G$ reaction size at 2 months of age

\begin{tabular}{|c|c|c|c|c|c|c|}
\hline $\begin{array}{l}\text { RCT (years with } \\
\text { inclusions) }\end{array}$ & $\begin{array}{l}\text { Small reaction } \\
\%(n / N)\end{array}$ & $\begin{array}{l}\text { Medium reaction } \\
\%(\mathrm{n} / \mathrm{N})\end{array}$ & $\begin{array}{l}\text { Large reaction } \\
\%(n / N)\end{array}$ & $\begin{array}{l}\text { Had } B C G \\
\text { reaction } \\
\%(n / N)\end{array}$ & $\begin{array}{l}\text { No } B C G \text { reaction } \\
\%(n / N)\end{array}$ & Total \\
\hline \multicolumn{7}{|l|}{ RCTs I-III (2002-2013) } \\
\hline BCG-Denmark & $3.9(38 / 978)$ & $3.3(18 / 552)$ & $2.4(13 / 549)$ & $3.3(69 / 2079)$ & $7.1(8 / 112)$ & $3.5(77 / 2191)$ \\
\hline \multicolumn{7}{|l|}{ RCT IV (2013-2017) } \\
\hline $\begin{array}{l}\text { BCG-Denmark (2013- } \\
\text { 2016) }\end{array}$ & $2.6(13 / 500)$ & $2.0(9 / 458)$ & $0.0(0 / 509)$ & $1.5(22 / 1467)$ & $0.0(0 / 30)$ & $1.5(22 / 1497)$ \\
\hline BCG-Japan (2016-2017) & $3.9(4 / 102)$ & $1.0(1 / 107)$ & $0.0(0 / 142)$ & $1.4(5 / 351)$ & $0.0(0 / 11)$ & $1.4(5 / 362)$ \\
\hline \multicolumn{7}{|l|}{$\begin{array}{l}\text { RCT V phase I* (2014- } \\
\text { 2016) }\end{array}$} \\
\hline BCG-Denmark & $1.6(2 / 126)$ & $0.6(1 / 168)$ & $0.4(1 / 237)$ & $0.8(4 / 531)$ & $0.0(0 / 3)$ & $0.8(4 / 534)$ \\
\hline BCG-Russia & $0.8(2 / 240)$ & $0.0(0 / 163)$ & $0.0(0 / 85)$ & $0.4(2 / 488)$ & $4.6(1 / 22)$ & $0.6(3 / 510)$ \\
\hline \multicolumn{7}{|l|}{$\begin{array}{l}\text { RCT V phase II* }(2016- \\
2017)\end{array}$} \\
\hline BCG-Japan & $1.0(1 / 103)$ & $0.0(0 / 128)$ & $0.4(1 / 227)$ & $0.4(2 / 458)$ & $8.3(1 / 12)$ & $0.6(3 / 470)$ \\
\hline BCG-Russia & $2.4(4 / 164)$ & $0.8(1 / 134)$ & $0.0(0 / 132)$ & $1.2(5 / 430)$ & $0.0(0 / 18)$ & $1.1(5 / 448)$ \\
\hline \multicolumn{7}{|l|}{ Total (2002-2017) } \\
\hline BCG-Denmark (RCTs I-V) & $3.3(53 / 1604)$ & $2.4(28 / 1178)$ & $1.1(14 / 1295)$ & $2.3(95 / 4077)$ & $5.5(8 / 145)$ & $2.4(103 / 4222)$ \\
\hline BCG-Japan (RCTs IV-V) & $2.4(5 / 205)$ & $0.4(1 / 225)$ & $0.3(1 / 369)$ & $0.9(7 / 809)$ & $4.4(1 / 23)$ & $1.0(8 / 832)$ \\
\hline BCG-Russia (RCT V) & $1.5(6 / 404)$ & $0.3(1 / 297)$ & $0.0(0 / 217)$ & $0.8(7 / 918)$ & $2.5(1 / 40)$ & $0.8(8 / 958)$ \\
\hline RCTs I-V, all strains & $2.9(64 / 2213)$ & $1.8(30 / 1710)$ & $0.8(15 / 1881)$ & $1.9(109 / 5804)$ & $4.8(10 / 208)$ & $2.0(119 / 6012)$ \\
\hline
\end{tabular}

RCT IV and RCT V recruited neonates up to September and October 2017, respectively, and follow-up procedures for the last recruited infants were completed one year later.

${ }^{*}$ RCT V featured two phases: one randomising infants to BCG-Denmark versus BCG-Russia (phase I) and one randomising infants to BCG-Japan versus BCG-Russia (phase II).

$\mathrm{BCG}$, Bacille Calmette-Guérin; RCT, randomised controlled trial; RR, risk ratio. 
Table 4 Mortality risk between 2 and 12 months by 2-month BCG reaction status and size

\begin{tabular}{|c|c|c|c|}
\hline BCG skin reaction & $\begin{array}{l}\text { 2-12 month mortality rate } \\
\text { per } 100 \text { Pyrs (deaths/Pyrs) (N) }\end{array}$ & Crude* MRR (95\% Cl) & $\begin{array}{l}\text { Adjusted† MRR (95\% } \\
\text { CI) }\end{array}$ \\
\hline \multicolumn{4}{|c|}{ Mortality risk by BCG reaction status } \\
\hline No reaction & $6.3(10 / 159)(208)$ & Ref. & Ref. \\
\hline Has reaction & $2.4(109 / 4504)(5804)$ & $0.48(0.25$ to 0.93$)$ & 0.49 (0.26 to 0.95$)$ \\
\hline \multicolumn{4}{|c|}{ Mortality risk by $B C G$ reaction size } \\
\hline Small reaction & $3.8(64 / 1704)(2213)$ & Ref. & Ref. \\
\hline Medium reaction & $2.3(30 / 1333)(1710)$ & 0.68 (0.44 to 1.05$)$ & $0.69(0.44$ to 1.07$)$ \\
\hline Large reaction & $1.0(15 / 1466)(1881)$ & 0.33 (0.19 to 0.58$)$ & 0.35 (0.20 to 0.63$)$ \\
\hline \multicolumn{4}{|c|}{ BCG-Denmark (RCTs I-V) } \\
\hline \multicolumn{4}{|c|}{ Mortality risk by $B C G$ reaction size } \\
\hline Small reaction & $4.3(53 / 1229)(1604)$ & Ref. & Ref. \\
\hline Medium reaction & $3.1(28 / 912)(1178)$ & $0.80(0.50$ to 1.26$)$ & $0.82(0.51$ to 1.31$)$ \\
\hline Large reaction & $1.4(14 / 1014)(1295)$ & 0.39 (0.22 to 0.71$)$ & 0.43 (0.23 to 0.78$)$ \\
\hline \multicolumn{4}{|c|}{ BCG-Japan (RCTs IV-V) } \\
\hline \multicolumn{4}{|c|}{ Mortality risk by BCG reaction size } \\
\hline Small reaction & $3.1(5 / 159)(205)$ & Ref. & Ref. \\
\hline Medium reaction & $0.5(1 / 184)(235)$ & $0.18(0.02$ to 1.55$)$ & $0.17(0.02$ to 1.47$)$ \\
\hline Large reaction & $0.4(1 / 283)(369)$ & 0.12 (0.01 to 1.03$)$ & $0.11(0.01$ to 0.96$)$ \\
\hline \multicolumn{4}{|c|}{ BCG-Russia (RCT V) } \\
\hline \multicolumn{4}{|c|}{ Mortality risk by $B C G$ reaction size } \\
\hline Small reaction & $1.9(6 / 316)(404)$ & Ref. & Ref. \\
\hline Medium reaction & $0.4(1 / 238)(297)$ & 0.22 (0.03 to 1.86$)$ & 0.19 (0.02 to 1.59$)$ \\
\hline Large reaction & $0.0(0 / 169)(217)$ & - & - \\
\hline
\end{tabular}

${ }^{*}$ Cox proportional hazards survival analysis (unadjusted, stratified by RCT), comparing mortality risk by reaction status (yes/no) and by BCG reaction size. †Cox proportional hazards survival analysis by BCG reaction status (has reaction versus no reaction, small versus medium and small versus large reaction size) adjusted for maternal MUAC, year and 2-month reaction assessor, stratified by study, providing the aMRR estimate.

BCG, Bacille Calmette-Guérin; MRR, mortality rate ratio; Pyrs, person-years.

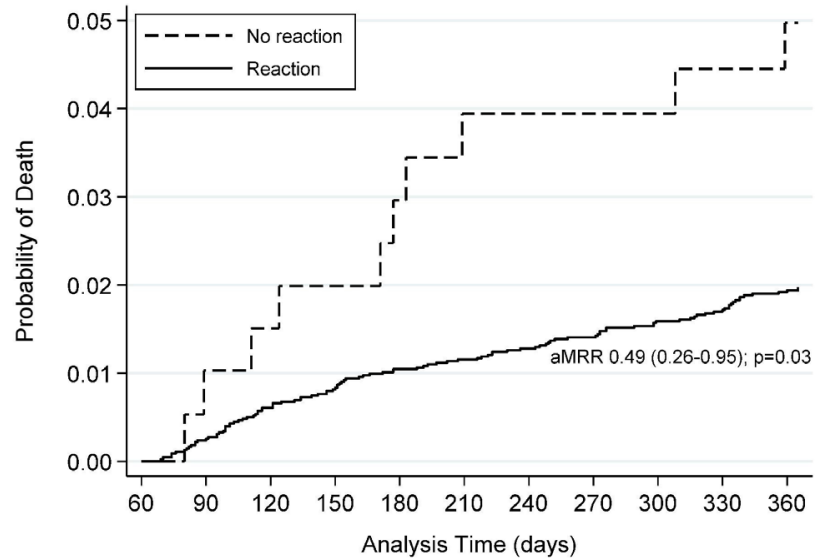

Number at risk

$\begin{array}{llllllllllll}\text { No reaction } & 6 & 200 & 205 & 203 & 200 & 194 & 191 & 189 & 188 & 186 & 182\end{array}$

Figure 3 Kaplan-Meier curve of cumulative deaths up to 1 year of age among infants with a Bacille Calmette-Guérin reaction versus no reaction. The statistical analysis is a reaction/no reaction Cox proportional hazards regression model with non-reactors as baseline, adjusted for maternal mid upper-arm circumference, year and 2-month reaction assessor, stratified by study, providing the adjusted mortality rate ratio (aMRR) estimate.

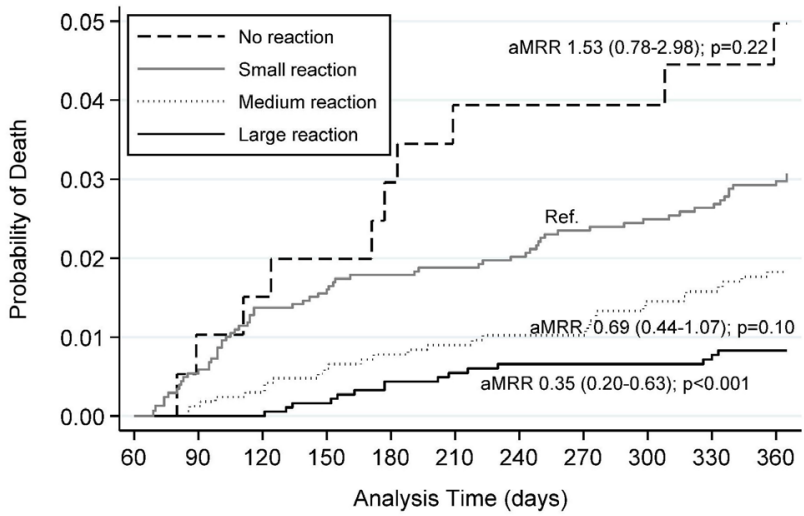

Number at risk

$\begin{array}{llllllllllll}\text { Large reaction } & 14 & 1832 & 1853 & 1836 & 1816 & 1787 & 1775 & 1765 & 1749 & 1740 & 1726\end{array}$ $\begin{array}{llllllllllll}\text { Medium reaction } & 25 & 1668 & 1683 & 1659 & 1651 & 1626 & 1612 & 1604 & 1594 & 1584 & 1570\end{array}$ $\begin{array}{cccccccccccc}\text { No reaction } & 6 & 200 & 205 & 203 & 200 & 194 & 191 & 189 & 188 & 186 & 182\end{array}$

Figure 4 Kaplan-Meier curve of cumulative deaths up to 1 year of age by Bacille Calmette-Guérin reaction size. All statistical analyses were conducted using Cox proportional hazards regression models with infants with small reactions as baseline, adjusted for maternal mid upper-arm circumference, year and 2-month reaction assessor, stratified by study, providing the aMRR estimate. aMRR, adjusted mortality rate ratio 


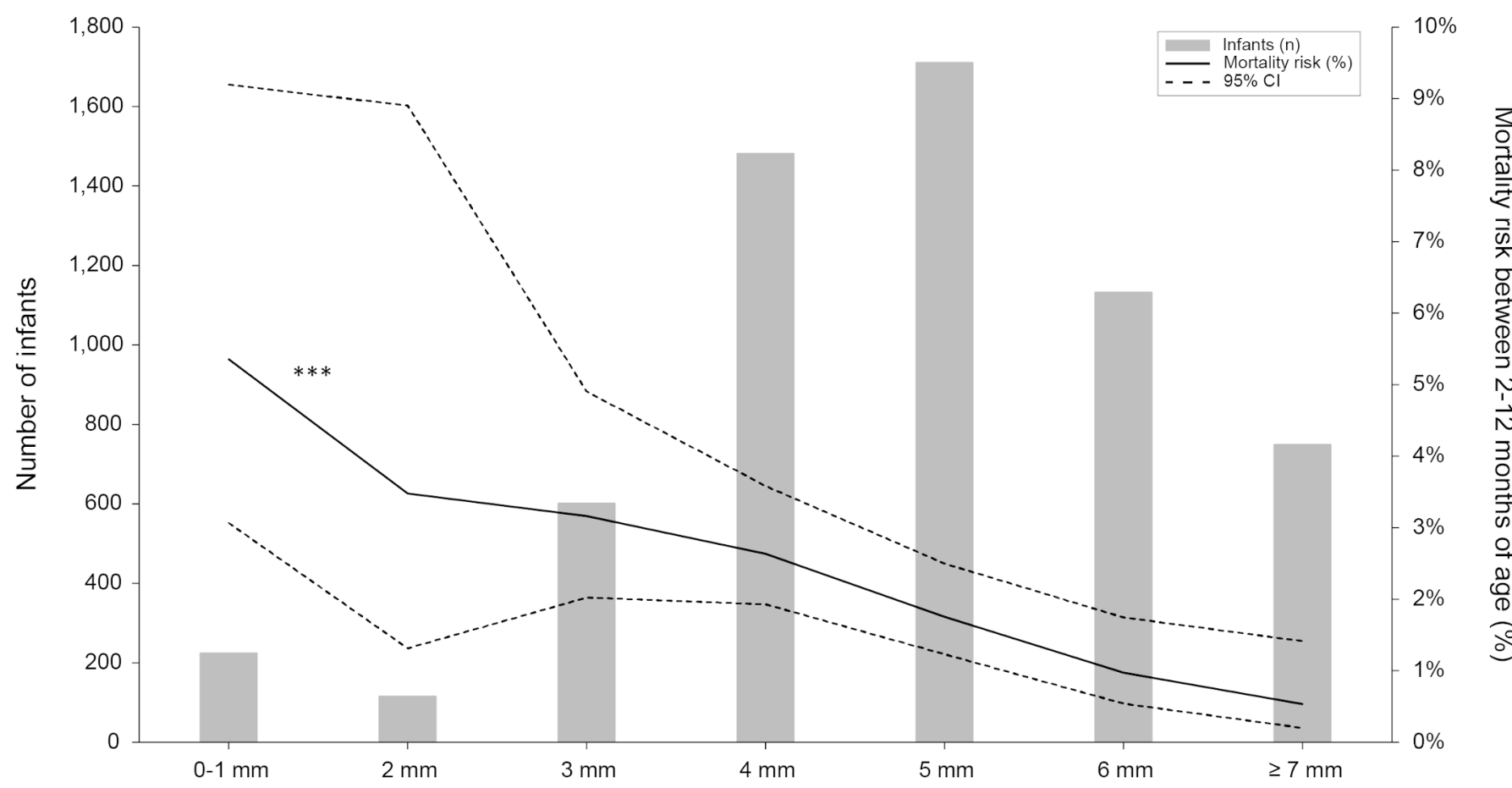

Reaction size at 2 months $(\mathrm{mm})$

Figure 5 Mortality risk by BCG reaction size in millimetres. ${ }^{* \star *} \mathrm{P}$ for trend $<0.001$ (logistic regression). $95 \%$ Cls (dotted lines) were calculated by logistic regression. Reaction sizes were calculated as the height plus the width of the reaction, divided by two. Reaction sizes displayed in this figure were rounded upwards: for example, a reaction size of $3.5 \mathrm{~mm}$ (width $4.0 \mathrm{~mm}$ and height $3.0 \mathrm{~mm}$ ) was rounded to $4 \mathrm{~mm}$. BCG, Bacille Calmette-Guérin

between BCG strains (table 4) and there was a consistent trend of decreasing mortality with increasing reaction size ( $\mathrm{p}$ for trend $<0.001$, figure 5 ). There were no significant differences between the sexes (online supplemental table 2). The mortality effects were consistent across the RCTs, with the exception that RCT IV had no deaths in the non-reactor group (online supplemental table 3 ).

Most deaths were caused by infection, $76 \%(87 / 114)$, and the reactor/non-reactor aMRR for infectious conditions was $0.53(0.24-1.17)$ (online supplemental table $4)$. By reaction size, the infection aMRR for large versus small reactions was $0.32(0.16-0.65)$. Our surveillance registered one death from $\mathrm{TB}$ in the cohort (online supplemental table 4).

There were no differences in reaction rates between ethnic groups, by maternal BCG scar status, by birth weight group (low weight vs normal weight) or by neonatal vitamin A supplementation status (table 5). The median maternal age was 26 years for reactors versus 23 years for non-reactors $(\mathrm{p}=0.001)$ and the median maternal MUAC was $260 \mathrm{~mm}$ for reactors and $250 \mathrm{~mm}$ for non-reactors $(\mathrm{p}<0.01)$ (table 5$)$. Due to the time trend of declining mortality across the RCTs and the possibility of differences in reaction assessments by the 2-month reaction assessor, the aMRRs presented were stratified by RCT and adjusted for maternal MUAC, year and 2-month reaction assessor. Our analyses were robust to adjustment for Caesarean section, maternal age and infant MUAC by 2 months of age.
Place of inclusion, vaccinator and size of the post-vaccination weal

The RR for no-reaction was 2.43 (1.50-3.92) for inclusion at health centres versus HNSM (RCTs I-III, table 5). In RCTs III-V, the RR for no-reaction was 1.83 (1.25-2.67) for vaccinator 1 versus vaccinator 2 . The mean size of the post-vaccination weal (measured only in RCT V) was $4.3 \mathrm{~mm}$ for vaccinator 1 and $4.5 \mathrm{~mm}$ for vaccinator 2 $(\mathrm{p}<0.001)$. The RR for no-reaction was $3.01(1.44-6.29)$ for medium $(\geq 4 \mathrm{~mm},<5 \mathrm{~mm}$ ) versus large $(\geq 5 \mathrm{~mm})$ postvaccination weal size and $5.91(2.62-13.3)$ for small $(<4$ $\mathrm{mm}$ ) versus large weal (table 6 ). There tended to be more medium and large-sized reactions and fewer small reactions associated with large weals (table 6).

\section{BCG strain (RCT V)}

For BCG-Denmark, 1\% (3/534) of infants did not develop a reaction versus $4 \%$ (22/510) for BCG-Russia. Hence, the RR for no-reaction was 7.68 (2.31-25.5) for BCG-Russia versus BCG-Denmark (table 6). Large reactions represented 44\% (237/534) of reactions for BCG-Denmark and 17\% (85/510) for BCG-Russia, the RR for large reaction being 0.38 (0.300.47 ) for BCG-Russia versus BCG-Denmark (table 6).

In phase II, 3\% (12/470) of infants vaccinated with BCGJapan did not develop a reaction versus $4 \%(18 / 448)$ for BCG-Russia, the RR for no-reaction being 1.57 (0.77-3.23) for BCG-Russia versus BCG-Japan. Large reactions represented 48\% (227/470) of reactions for BCG-Japan and 29\% 
Table 5 Determinants for having a BCG reaction at 2 months of age

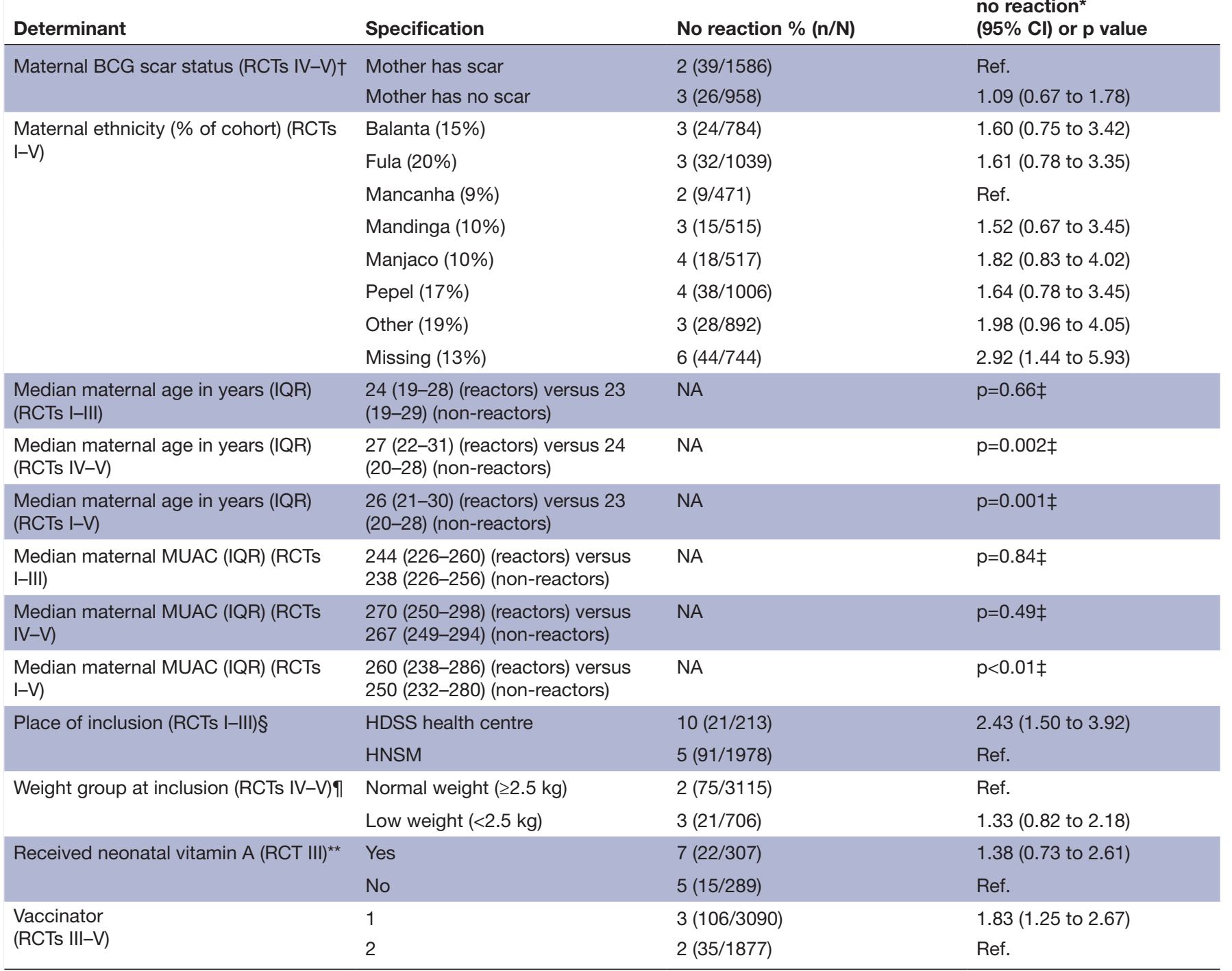

*Binomial regression, adjusted for RCT.

tWithin a subgroup of 2544 infants included in RCTs IV-V, maternal BCG reaction assessments were performed at inclusion.

$\ddagger$ Kruskal-Wallis $\chi^{2}$ test with ties.

§Analysis comparing RCT I-III neonates since all infants in RCT IV-V were included at HNSM. At HNSM, vaccinator 1 and 2 performed all vaccinations while vaccinations at the HDSS health centres were performed by Bandim Health Project assistants that less frequently performed BCG vaccinations.

I|Analysis within studies IV-V which recruited both normal birth weight and low birth weight infants.

${ }^{* \star}$ RCT II was designed as a 2-by-2 factorial RCT with a subgroup of neonates randomised to receive either 25000 International Units of NVAS or placebo, as well as either early-BCG or no-BCG.

BCG, Bacille Calmette-Guérin; MUAC, mid-upper-arm circumference; NA, not applicable; RCT, randomised controlled trial.

(132/448) for BCG-Russia, the RR for large reaction being 0.61 (0.51-0.72) for BCG-Russia versus BCG-Japan (table 6).

\section{Evaluation of the correlation between cytokine responses and skin reaction by 4 weeks}

In RCT III, a subgroup of 224 BCG-vaccinated infants had their BCG skin reaction assessed at a 4-week homevisit where a blood sample was obtained for immunological assessment. The 4-week BCG reaction prevalence was 92\% (205/224) and having a 4-week BCG reaction was associated with a higher monocyte cell count, $1760 / \mu \mathrm{L}$ (1340-2330) for reactors versus $1240 / \mu \mathrm{L}$ (950-1830) for non-reactors $(\mathrm{p}=0.02)$. The neutrophil cell count was $2070 / \mu \mathrm{L}(1450-3010)$ for reactors versus $1850 / \mu \mathrm{L}$ (790-2610) for non-reactors $(\mathrm{p}=0.19)$. BCG reactors had higher responses to stimulation with PPD, especially IFN- $\gamma \quad(G M R=4.62(1.70-12.4))$, when compared with non-reactors (online supplemental figure 1A, table 5). A comparable pattern was seen after stimulation with heterologous TLR agonists and the pattern was strongest for the TLR-8 agonist CL075 (IFN- $\gamma$ : GMR=2.20 (1.054.62)), whereas both unstimulated and PMA-stimulated responses were generally higher among non-reactors 


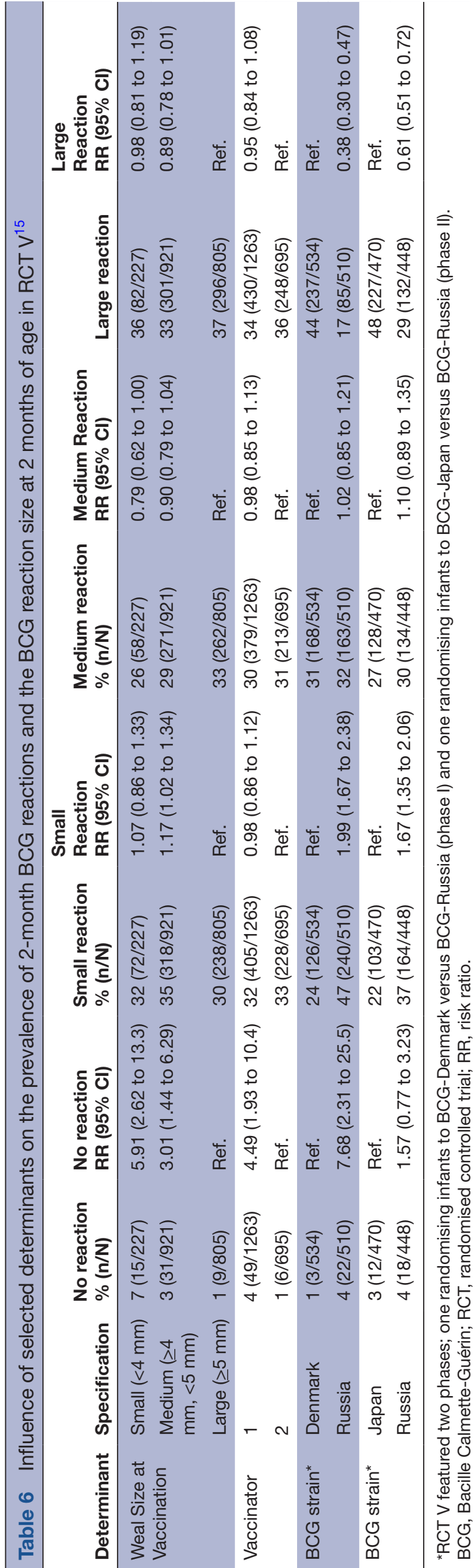

(PMA: IFN- $\gamma$ : GMR $=0.80 \quad(0.75-0.87)$; unstimulated: IL-1 $\beta:$ GMR=0.40 (0.17-0.95), IL-6: GMR=0.33 (0.14$0.79)$, TNF- $\alpha$ : GMR $=0.51 \quad(0.30-0.87), \mathrm{IL}-17:$ GMR $=0.44$ $(0.20-0.95)$ and IL-10: GMR $=0.45(0.21-0.96))$. Among all stimulus-cytokine combinations, a larger reaction was generally associated with higher cytokine responses (online supplemental figure 1B,C).

\section{DISCUSSION}

Having an early BCG reaction by 2 months of age was associated with a substantial reduction in 2-12 months mortality. The larger the reaction, the more pronounced was the beneficial effect on survival. This association was consistent across five RCTs and three BCG strains and protection was mainly against deaths caused by infectious diseases.

The main determinants for developing a BCG reaction were related to the quality of the BCG vaccination and the BCG strain used; BCG-Denmark and BCG-Japan were associated with higher reaction rates and larger reaction sizes than BCG-Russia.

By 4 weeks of age, both specific and heterologous cytokine responses were more pronounced among BCG reactors, and even more so in those with large 4-week reactions.

\section{Strengths and weaknesses}

Previous observational studies have examined infants vaccinated mainly during the first months of life rather than in the first week, and the BCG reaction assessments have mostly taken place later in infancy, focusing on the formation of the final BCG scar. Since mortality is higher earlier in life and BCG-at-birth is the recommended policy, and because there is less pre-vaccination exposure to environmental mycobacteria for those vaccinated at birth, it is important to evaluate the effects of BCG provided as close to birth as possible and the associated BCG skin reactions.

While birth weight was not a determinant for developing a BCG reaction, reactors had better 2-month anthropometric indices than non-reactors. Among reactors, larger reaction size was associated with higher 2-month MUAC indicating that infants with larger reaction sizes were or had become healthier. We considered the MUAC a mediator of the overall health effect of adequate BCG vaccination and our analyses were robust to adjustments for factors known to affect mortality.

Differences in classification of BCG reactions between different reaction assessors could have affected our results, but our analyses were robust to adjustment for the reaction assessor. Our analyses were stratified by RCT and revealed a consistent pattern of reduced mortality associated with both having a reaction and the reaction size across the trials.

The relative effect of having a large reaction was greatest in the two most recent trials, despite a lower overall mortality, a higher BCG reaction prevalence and more large reactions than in RCTs I-III. This is important because it indicates that BCG reaction sizes are important predictors of survival also in situations where mortality is 
relatively low and vaccination quality is high. The trend of reduced mortality associated with having an early BCG reaction and the reaction size, which was consistent across the five trials spanning 17 years, was robust to adjustment for RCT and calendar year, making confounding by chronology unlikely.

We only had cytokine data by 4 weeks of age, and could thus only conclude that cytokine production by 4 weeks of age correlated with BCG reaction characteristics at 4 weeks of age, providing only circumstantial evidence for BCG reactions being correlated with increased cytokine responses to PPD and heterologous stimuli by 2 months of age.

Our surveillance detected only one death due to TB and it is thus unlikely that specific protection against TB played a substantial role in the observed effects.

\section{Interpretation}

All available studies have reported a beneficial effect on all-cause mortality of developing a BCG scar after BCG vaccination, but there is a remarkable variation in the BCG scar prevalence ranging from 52\% (18 535/35 416) in a large observational study from rural Bissau ${ }^{11}$ to the $97 \%(5804 / 6012)$ reaction prevalence in the present study. This indicates that the beneficial effects on mortality are not merely caused by healthier infants responding better to vaccination. Rather, as corroborated in the present study, it is the quality of vaccination (postvaccination weal size, vaccination by trained personnel at a major hospital) and the BCG strain administered that are the main determinants for developing a reaction and the size of the reaction.

We found that BCG-Russia is associated with fewer and smaller BCG reactions by 2 months of age. Previous BHP studies and a study from Uganda have indicated that BCGRussia produces fewer BCG scars. ${ }^{14} 27-29$ In a small RCT from Australia, BCG-Russia was associated with smaller skin reactions and less favourable immune responses ${ }^{17}$ and BCG-Russia was furthermore associated with fewer tuberculin skin test responses in the randomised comparisons with both BCG-Japan and BCG-Denmark in RCT $\mathrm{V}^{15}$ A large retrospective study from Kazakhstan reported that while BCG-Russia provided some protection against TB when compared with unvaccinated infants, BCGJapan was associated with the highest protective efficacy against TB. ${ }^{30}$ The available data thus indicates that BCGDenmark and BCG-Japan are more immunogenic than BCG-Russia. These findings might help explain why two recent RCTs providing BCG-Russia versus no BCG did not show an effect of BCG-Russia on the all-cause neonatal mortality. ${ }^{31}$ Other major BCG strains such as BCGBulgaria (genetically identical to BCG-Russia) and BCGGreenSignal (genetically identical to BCG-Denmark) remain to be evaluated.

BCG is a strong immunostimulant ${ }^{5}$ and a study in mice has underlined the importance of vaccination technique, indicating that access of BCG to the bone marrow changes the transcriptional landscape of haematopoietic stem cells and multipotent progenitors, inducing immune training. ${ }^{32}$ Similarly, a recent analysis in humans indicated that BCG induces transcriptomic myeloid priming of the haematopoietic stem and progenitor cell compartment, which induces transcription factors connected to myeloid cell function. This resulted in elevated granulocyte numbers and induced lasting innate immune system memory. ${ }^{33}$ Such biological effects depending on BCG's access to the bone marrow are likely influenced by the absolute number of viable mycobacteria deposited within the host, which are determined by the BCG strain, injection technique and the dose injected (postvaccination weal size). A recent laboratory study has elaborated the differences between BCG strains in terms of the content of colony-forming units and the percentage of live versus dead bacteria. ${ }^{34}$ The marked in vitro differences in viability between strains reported, which favour BCG-Denmark and BCG-Japan over BCG-Russia, provide a plausible biological explanation to our epidemiological findings. Large BCG reactions thus likely represent a well-trained innate immune system. We found that having an early reaction is accompanied with more innate immune training in vitro, which have been associated with reduced viral and parasitic load after challenge in other studies. ${ }^{35}{ }^{36}$ Intriguingly, early reactions were at the same time associated with less reactivity in the unstimulated samples, that is, in steady state, possibly reflecting less infection/inflammation in infants with an early BCG reaction.

\section{Policy implications}

It is paramount to introduce measures that eliminate known logistic barriers to vaccination ${ }^{37} 38$ so that BCG can be provided shortly after birth to produce high BCG reaction rates. We demonstrate that feasible targets are 2-month BCG reaction rates of $\geq 96 \%$ for BCG-Russia and $\geq 97 \%$ for BCG-Japan and BCG-Denmark, with $30 \%$ of early reactions being $\geq 6.0 \mathrm{~mm}$. BCG reactions should be surveyed by 2 months of age to detect immunisation failures, weak BCG strains and batch-to-batch variations that are known to occur within strains. ${ }^{39}$ Observational studies from rural Guinea-Bissau has reported that the 1-month BCG vaccination coverage was just $38 \%$ and among 35 416 BCG-vaccinated children under 5 years of age, the BCG scar prevalence was just 52\%. ${ }^{11} 38$ Similarly, a study from 2009 reported that the neonatal BCG vaccination coverage in sub-Saharan Africa is below 50\%, despite the WHO recommendation of BCG-at-birth. ${ }^{40}$ Updated data on vaccination coverage and early BCG reaction prevalence is needed, however, to fully assess the current impact of early BCG vaccination and the potential impact of improved vaccination policies. There seems to be a vast potential for reducing early-life mortality by ensuring adequate training of vaccinators, a high neonatal BCG coverage and surveillance of subsequent BCG reactions. Addressing immunisation failures by providing revaccination to non-reactors might also be an untapped lowcost public health measure that could both improve TB 
protection and reduce all-cause mortality. This could be performed in conjunction with the health centre contact occurring at 14 weeks of age, when the third dose of pentavalent vaccine is provided. As an added benefit, providing BCG at this time might remedy the previously reported detrimental NSEs on overall survival related to pentavalent vaccination. ${ }^{41}$ To enhance beneficial NSEs, the use of immunogenic BCG strains is to be encouraged.

\section{CONCLUSION}

Our analysis corroborated that developing a BCG reaction is associated with a substantial survival benefit and extended these findings, by showing that having a skin reaction already by 2 months of age and the reaction size were associated with substantial additional benefits. Compared with BCG-Russia, BCG-Denmark and BCGJapan induce more reactions that are also larger. This indicates a long-term association between adequate early vaccination with specific BCG strains, subsequent skin reaction kinetics, and survival.

Given the public health implications, it is urgent that data from other settings are examined to test whether our findings can be replicated. With little additional costs, an increased focus on timely BCG vaccination, immunogenic BCG strains and vaccination quality could save many infant lives globally, given that BCG is administered to $>120$ million infants per year.

\author{
Author affiliations \\ ${ }^{1}$ Bandim Health Project, Institute of Clinical Research, Uni. Southern Denmark and \\ Odense University Hospital, Odense, Denmark \\ ${ }^{2}$ Bandim Health Project, INDEPTH Network, Bissau, Guinea-Bissau \\ ${ }^{3}$ Research Centre for Vitamins and Vaccines, Copenhagen, Denmark \\ ${ }^{4}$ Department of Internal Medicine, Radboud Centre for Infectious Diseases, \\ Radboud University Medical Centre, Nijmegen, The Netherlands \\ ${ }^{5}$ Public Health Agency of Sweden, Solna, Sweden \\ ${ }^{6}$ Institution for Translational Medicine, Lund University, Malmö, Sweden \\ ${ }^{7}$ Experimental and Translational Immunology, Department of Health Technology, \\ Technical University of Denmark, Lyngby, Denmark \\ ${ }^{8}$ Danish Institute of Advanced Science, University of Southern Denmark, Odense, \\ Denmark
}

Twitter Frederik Schaltz-Buchholzer @FSBuchholzer and Christine Stabell Benn @StabellBenn

\section{Acknowledgements The authors wish to thank the mothers and infants who participated in the study.}

Contributors CSB and PA were the principal investigators and guarantors of the five RCTs. MB assisted in conceptualising the paper and conducted a systematic PubMed search of articles evaluating BCG reactions. MB furthermore conducted the analyses of cytokine responses assisted by KJJ, who was responsible for the blood sample collection within RCT III and who also analysed the samples in the laboratory and supervised RCT III data collection procedures. AR supervised RCT I-II. MB-A supervised RCT IV and helped plan RCT V with CSB, PA and FS-B. MKS assisted in the data cleaning and assessment of verbal autopsies within studies IV-V. IM was responsible for the data collection and data entry within all five RCTs. FS-B serendipitously conceived the idea that the BCG reaction size might affect infant health after vaccinating his daughter Mettezinha with BCG. FSB unified and cleaned the BCG reaction data from the five RCTs in one database, supervised RCTs III-V, conducted the main data analysis and wrote the first draft of the paper. All authors contributed to and approved the final paper.

Funding RCT II was funded by the European Union (ICA4-CT-2002-10053), the March of Dimes, and the Danish National Research Foundation. RCT III was supported by The European Research Council (starting grant
ERC-2009-StG-243149, which also funded IM and CSB); the Novo Nordisk Foundation (research professorship grant to PA); the Danish National Research Foundation (grant DNRF108 to the Research Centre for Vitamins \& Vaccines); DANIDA, European Union FP7, and OPTIMUNISE (grant Health-F3-2011-261 375 to the Bandim Health Project). RCT IV received support from the Karen Elise Jensen Foundation and RCTs IV-V were supported by The Danish National Research Foundation (DNRF) [grant number DNRF108]. DNRF and the University of Southern Denmark funded PhD scholarships to FS-B and MB. Bandim Health Project has previously received support by the Danish International Development Agency (DANIDA).

Disclaimer The funding agencies had no influence on the study design, data collection, analysis, interpretation or writing of the manuscript, nor the decision to submit the paper for publication. The authors had full access to all study data and bears the responsibility for their analysis and the decision to submit for publication.

\section{Competing interests None declared.}

Patient and public involvement Patients and/or the public were not involved in the design, or conduct, or reporting, or dissemination plans of this research.

\section{Patient consent for publication Not required.}

Ethics approval Original study protocols were approved by the Guinean Ministry of Health's Research Coordination Committee and the Central Danish Ethical Committee, and the Danish National Committee on Health Research Ethics had given its consultative approvals. Informed consent was obtained for all participants and the trials were conducted in accordance with the Declaration of Helsinki ethical standards. BHP offered free medical consultations and essential medicine to all infants accessed for eligibility. Participants could request to leave a trial at any given time.

Provenance and peer review Not commissioned; externally peer reviewed.

Data availability statement Data are available upon request. Deidentified participant data with a data dictionary can be shared after approval of a datasharing proposal sent to Professor Christine Stabell Benn (cbenn@health.sdu.dk).

Open access This is an open access article distributed in accordance with the Creative Commons Attribution Non Commercial (CC BY-NC 4.0) license, which permits others to distribute, remix, adapt, build upon this work non-commercially, and license their derivative works on different terms, provided the original work is properly cited, appropriate credit is given, any changes made indicated, and the use is non-commercial. See: http://creativecommons.org/licenses/by-nc/4.0/.

\section{ORCID iDs}

Frederik Schaltz-Buchholzer http://orcid.org/0000-0001-7643-8322

Mike Berendsen http://orcid.org/0000-0001-9516-6190

Kristoffer Jarlov Jensen http://orcid.org/0000-0001-9687-5706

Morten Bjerregaard-Andersen http://orcid.org/0000-0002-4128-5714

Marcus Kjær Sørensen http://orcid.org/0000-0002-5775-8854

Peter Aaby http://orcid.org/0000-0001-8331-1389

Christine Stabell Benn http://orcid.org/0000-0001-6102-3810

\section{REFERENCES}

1 Bottai D, Brosch R. The BCG strain pool: diversity matters. Mol Ther 2016;24:201-3.

2 Higgins JPT, Soares-Weiser K, López-López JA, et al. Association of BCG, DTP, and measles containing vaccines with childhood mortality: systematic review. BMJ 2016;355:i5170.

3 Biering-Sørensen S, Aaby P, Lund N, et al. Early BCG-Denmark and neonatal mortality among infants weighing $<2500 \mathrm{~g}$ : a randomized controlled trial. Clin Infect Dis 2017;65:1183-90.

4 Schaltz-Buchholzer F, Biering-Sørensen S, Lund N, et al. Early BCG vaccination, hospitalizations, and hospital deaths: analysis of a secondary outcome in 3 randomized trials from Guinea-Bissau. Infect Dis 2019;219:624-32.

5 Benn CS, Netea MG, Selin LK, et al. A small jab - a big effect: nonspecific immunomodulation by vaccines. Trends Immunol 2013;34:431-9.

6 Kleinnijenhuis J, Quintin J, Preijers F, et al. Bacille Calmette-Guerin induces NOD2-dependent nonspecific protection from reinfection via epigenetic reprogramming of monocytes. Proc Natl Acad Sci U S A 2012;109:17537-42.

7 Garly M-L, Martins CL, Balé C, et al. BCG scar and positive tuberculin reaction associated with reduced child mortality in West Africa. A non-specific beneficial effect of BCG? Vaccine 2003;21:2782-90. 
8 Roth A, Gustafson P, Nhaga A, et al. BCG vaccination scar associated with better childhood survival in Guinea-Bissau. Int $J$ Epidemiol 2005;34:540-7.

9 Roth A, Sodemann M, Jensen H, et al. Tuberculin reaction, BCG scar, and lower female mortality. Epidemiology 2006;17:562-8.

10 Timmermann CAG, Biering-Sørensen S, Aaby P, et al. Tuberculin reaction and BCG scar: association with infant mortality. Trop Med Int Health 2015;20:1733-44.

11 Storgaard L, Rodrigues A, Martins C, et al. Development of BCG scar and subsequent morbidity and mortality in rural Guinea-Bissau. Clin Infect Dis 2015;61:950-9.

12 Berendsen MLT, Øland CB, Bles $\mathrm{P}$, et al. Maternal priming: Bacillus Calmette-Guérin (BCG) vaccine scarring in mothers enhances the survival of their child with a BCG vaccine scar. J Pediatric Infect Dis Soc 2020;9:166-72.

13 Benn CS, Roth A, Garly M-L, et al. BCG-scarring and improved child survival: a combined analysis of studies of BCG scarring. J Intern Med 2020. doi:10.1111/joim.13084. [Epub ahead of print: $16 \mathrm{Apr}$ 2020].

14 Roth A, Sodemann M, Jensen $\mathrm{H}$, et al. Vaccination technique, PPD reaction and BCG scarring in a cohort of children born in GuineaBissau 2000-2002. Vaccine 2005;23:3991-8.

15 Schaltz-Buchholzer F, Bjerregaard-Andersen M, Øland CB, et al. Early vaccination with BCG-Denmark or BCG-Japan versus BCG-Russia to healthy newborns in Guinea-Bissau: a randomized controlled trial. Clin Infect Dis 2019. doi:10.1093/cid/ciz1080. [Epub ahead of print: 03 Nov 2019].

16 Birk NM, Nissen TN, Ladekarl M, et al. The association between

Bacillus Calmette-Guérin vaccination (1331 SSI) skin reaction and subsequent scar development in infants. BMC Infect Dis 2017; $17: 540$

17 Ritz N, Dutta B, Donath S, et al. The influence of Bacille CalmetteGuerin vaccine strain on the immune response against tuberculosis: a randomized trial. Am J Respir Crit Care Med 2012;185:213-22.

18 Biering-Sørensen S, Aaby P, Napirna BM, et al. Small randomized trial among low-birth-weight children receiving Bacillus CalmetteGuérin vaccination at first health center contact. Pediatr Infect Dis J 2012;31:306-8.

19 Aaby P, Roth A, Ravn H, et al. Randomized trial of BCG vaccination at birth to low-birth-weight children: beneficial nonspecific effects in the neonatal period? J Infect Dis 2011;204:245-52.

20 U.S. National Library of Medicine. A randomized trial of providing BCG vaccination immediately. Available: https://clinicaltrials.gov/ct2/ show/NCT01989026 [Accessed 2 Jan 2019].

21 Guerin N, Bregere P. BCG vaccination. Child Trop 1992:72-6.

22 World Health Organization . BCG vaccine: WHO position paper, February 2018 - Recommendations. Vaccine 2018;36:3408-10.

23 Thysen SM, Fernandes M, Benn CS, et al. Cohort profile : Bandim Health Project's (BHP) rural Health and Demographic Surveillance System (HDSS)-a nationally representative HDSS in Guinea-Bissau. BMJ Open 2019;9:e028775.

24 Jensen KJ, Larsen N, Biering-Sørensen S, et al. Heterologous immunological effects of early BCG vaccination in low-birth-weight infants in Guinea-Bissau: a randomized-controlled trial. J Infect Dis 2015;211:956-67.

25 World Health Organization. Verbal autopsy standards: the 2012 WHO verbal autopsy instrument, 2012. Available: http://www.who.int/ healthinfo/statistics/WHO VA 2012 RC1_Instrument.pdf [Accessed 10 Oct 2019].
26 Uh H-W, Hartgers FC, Yazdanbakhsh M, et al. Evaluation of regression methods when immunological measurements are constrained by detection limits. BMC Immunol 2008;9:59.

27 Frankel H, Byberg S, Bjerregaard-Andersen M, et al. Different effects of BCG strains - A natural experiment evaluating the impact of the Danish and the Russian BCG strains on morbidity and scar formation in Guinea-Bissau. Vaccine 2016;34:4586-93.

28 Funch KM, Thysen SM, Rodrigues A, et al. Determinants of BCG scarification among children in rural Guinea-Bissau: a prospective cohort study. Hum Vaccin Immunother 2018;14:1-9.

29 Anderson EJ, Webb EL, Mawa PA, et al. The influence of BCG vaccine strain on mycobacteria-specific and non-specific immune responses in a prospective cohort of infants in Uganda. Vaccine 2012;30:2083-9.

30 Favorov M, Ali M, Tursunbayeva A, et al. Comparative tuberculosis (TB) prevention effectiveness in children of Bacillus Calmette-Guérin (BCG) vaccines from different sources, Kazakhstan. PLoS One 2012;7:e32567

31 Jayaraman K, Adhisivam B, Nallasivan S, et al. Two randomized trials of the effect of the Russian strain of Bacillus Calmette-Guérin Alone or with oral polio vaccine on neonatal mortality in infants weighing $<2000 \mathrm{~g}$ in India. Pediatr Infect Dis $J$ 2019;38:198-202.

32 Kaufmann E, Sanz J, Dunn JL, et al. BCG Educates hematopoietic stem cells to generate protective innate immunity against tuberculosis. Cell 2018;172:176-90.

33 Cirovic B, de Bree LCJ, Groh L, et al. BCG vaccination in humans elicits trained immunity via the hematopoietic progenitor compartment. Cell Host Microbe 2020;28:322-34.

34 Angelidou A, Conti M-G, Diray-Arce J, et al. Licensed Bacille Calmette-Guérin (BCG) formulations differ markedly in bacteria viability, RNA content and innate immune activation. Vaccine 2020;38:2229-40.

35 Arts RJW, Moorlag SJCFM, Novakovic B, et al. BCG vaccination protects against experimental viral infection in humans through the induction of cytokines associated with trained immunity. Cell Host Microbe 2018;23:89-100.

36 Walk J, de Bree LCJ, Graumans W, et al. Outcomes of controlled human malaria infection after BCG vaccination. Nat Commun 2019;10:874

37 Schaltz-Buchholzer F, Frankel HN, Benn CS. The real-life number of neonatal doses of Bacille Calmette-Guérin vaccine in a 20-dose vial. Glob Health Action 2017;10:1-4.

38 Thysen SM, Byberg S, Pedersen M, et al. BCG coverage and barriers to BCG vaccination in Guinea-Bissau: an observational study. BMC Public Health 2014;14:1037.

39 Biering-Sørensen S, Jensen KJ, Aamand SH, et al. Variation of growth in the production of the BCG vaccine and the association with the immune response. An observational study within a randomised trial. Vaccine 2015;33:2056-65.

40 Clark A, Sanderson C. Timing of children's vaccinations in 45 lowincome and middle-income countries: an analysis of survey data. Lancet 2009;373:1543-9.

41 Aaby P, Andersen A, Ravn H, et al. Co-administration of BCG and diphtheria-tetanus-pertussis (DTP) vaccinations may reduce infant mortality more than the WHO-schedule of BCG first and then DTP. A Re-analysis of demographic surveillance data from rural Bangladesh. EBioMedicine 2017;22:173-80. 